\title{
CARPHOLOGY by A Fo Ben
}

Pract Neurol 2010; 10: 368

\section{Stroke: top 10 risks in developing countries}

INTERSTROKE, a standardised case control study $(n=3000)$ looked for the most important stroke risk factors in low income countries. Hypertension was the most important (OR 2.64, 99\% Cl 2.26 to 3.08), with current smoking (OR 2.09) and waist to hip ratio (OR 1.65) close behind. Other high risk scores were for diet and physical activity, diabetes mellitus, alcohol, psychosocial stress, depression, cardiac causes and apolipoprotein $B: A 1$ ratio. Targeted interventions in the developing world to reduce blood pressure and smoking, and to promote physical activity and a healthy diet, are needed to reduce the global burden of stroke.

Lancet 2010;376:112-23.

\section{Thrombolysis: can't come too soon}

Early thrombolysis for acute ischaemic stroke is beneficial but delaying it may be hazardous. A large meta-analysis of eight placebo controlled trials $(n=2775)$ of intravenous recombinant tissue plasminogen activator showed most benefit (at 3 months) when alteplase was given at 0-90 min (OR 2.55, $95 \% \mathrm{Cl} 1.44$ to 4.52$)$ but only 1.34 (1.06-1.68) at 181-270 min and $1.22(0.92-1.61)$ at 271$360 \mathrm{~min}$. Beyond $4.5 \mathrm{~h}$, the risks outweighed benefits with a trend towards increased mortality. Despite the practical difficulty of urgent management of acute ischaemic stroke, stroke physicians must recognise the risk to delaying thrombolysis, even within the currently recommended time windows.

Lancet 2010;375:1695-703.

\section{Weight for IIH}

Idiopathic intracranial hypertension $(\mathrm{IIH})$ is eight times more common in the overweight or obese. Everyone knows weight reduction is good for IIH but there is no definite (randomised controlled trial) proof. A prospective non-randomised cohort study of women with
IIH $(n=25)$ showed that significant weight reduction (15.7 (SD 8.0) kg, p<0.001) was associated with falls in intracranial pressure (8.0 (SD 4.2) cm CSF, p<0.001), headache score, papilloedema, optic nerve sheath diameter and optical coherence tomography score. Do we still have to wait for a randomised controlled trial to prove the obvious? BMJ 2010;340:c2701.

\section{Cells that fire together, expire together}

Genetic studies that produce neither compellingly powerful results nor add to an understanding of the underlying biology, frustrate experts and outsiders alike. Therefore, the report that carrying a risk genotype-at three single nucleotide polymorphisms-increases depressed patients' risk of attempting suicide in their lifetime 4.5-fold ( $95 \% \mathrm{Cl} 2.1$ to $\left.9.8 ; p=4.7 \times 10^{-7}\right)$ is a welcome tonic. The finding was confirmed in two other ethnically stratified populations and focused on the neurotrophic receptorencoding gene NTRK2. Dysfunctional NTRK2 signalling appears to impact on neurotrophic factors, leading to altered neuronal survival and synaptic plasticity.

Arch Gen Psychiatry 2010;67:348-59.

\section{Balo's concentric sclerosis}

A 32-year-old woman with fever, slurred speech and right hemiparesis had rings of alternating intensity in the left parietal lobe on her MR brain scan. Balo's concentric sclerosis is a rare feature of a severe acute inflammatory demyelinating disorder. Jozsef Balo (1895-1979) was a Hungarian pathologist who described this first as leukoencephalitis periaxialis concentrica. Lancet 2010;376:189.

\section{Laser safety blind spot}

A previously healthy teenager developed central scotomas and reduced visual acuity (6/12) after shining a green diode laser pointer into his eyes. Fundoscopy showed foveal granularity; fluorescein angiography and optical coherence tomography confirmed retinal epithelial disturbance. His visual acuity returned to $6 / 6$ at 2 months. Laser pointers sold to the public must have laser power below $1 \mathrm{~mW}$ (British Standard) but their risks are poorly researched and their long term threats to vision unknown. BMJ 2010;340:1261.

A Fo Ben is always on the lookout for suitable Carphology titbits and comments on what has been included. Email the editorin-chief if you come across anything.
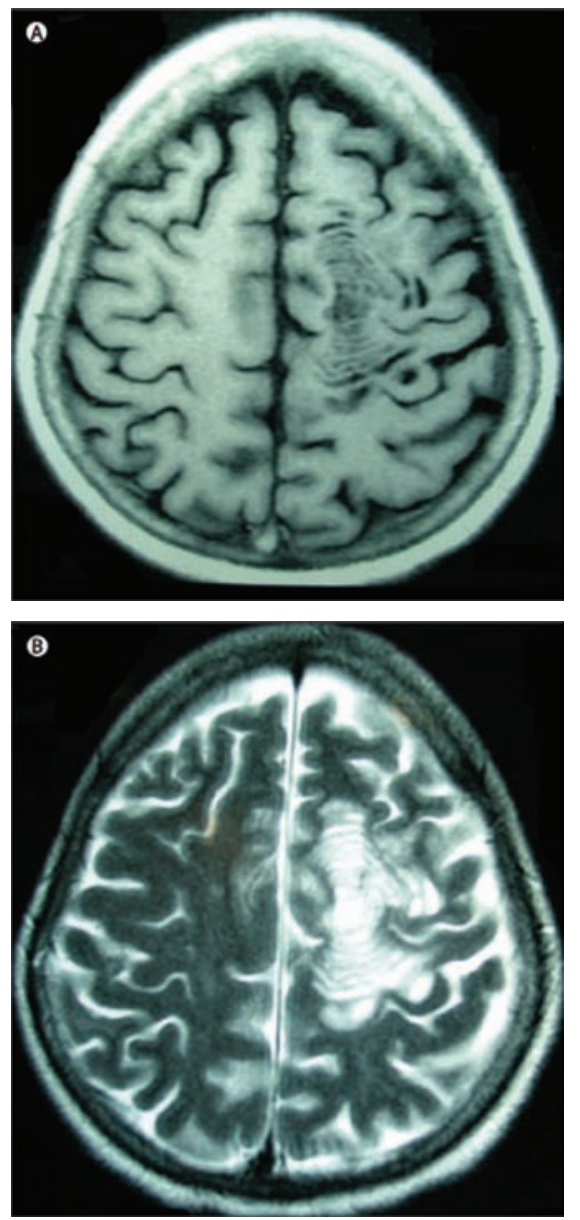

MR brain scan. (A) T1 weighted, (B) T2 weighted. (Reproduced with permission.) 